\title{
Review Article \\ Examining the Pathways Linking Chronic Sleep Restriction to Obesity
}

\author{
Christopher A. Magee, ${ }^{1}$ Xu-Feng Huang, ${ }^{2}$ Donald C. Iverson, ${ }^{1}$ and Peter Caputi ${ }^{3}$ \\ ${ }^{1}$ Illawarra Health and Medical Research Institute, University of Wollongong, Wollongong, NSW 2522, Australia \\ ${ }^{2}$ School of Health Sciences, University of Wollongong, Wollongong, NSW 2522, Australia \\ ${ }^{3}$ School of Psychology, University of Wollongong, Wollongong, NSW 2522, Australia
}

Correspondence should be addressed to Christopher A. Magee, cmagee@uow.edu.au

Received 21 August 2009; Revised 2 December 2009; Accepted 23 December 2009

Academic Editor: Eric Doucet

Copyright ( $) 2010$ Christopher A. Magee et al. This is an open access article distributed under the Creative Commons Attribution License, which permits unrestricted use, distribution, and reproduction in any medium, provided the original work is properly cited.

A growing number of studies have identified chronic sleep restriction as a potential risk factor for obesity. This could have important implications for how obesity is prevented and managed, but current understanding of the processes linking chronic sleep restriction to obesity is incomplete. In this paper, we examined some of the pathways that could underlie the relationship between chronic sleep restriction and obesity. This involved exploring some of the potential environmental, health, behavioral, and sociodemographic determinants of chronic sleep restriction, which require further investigation in this context. Three pathways that could potentially link chronic sleep restriction to obesity were then examined: (1) altered neuroendocrine and metabolic function, (2) impaired glucose regulation, and (3) waking behavior. The selected pathways linking chronic sleep restriction to obesity reviewed in this paper are presented in a schematic representation; this may be used to guide future research in this area. This area of research is important because it may lead to more effective interventions and strategies to combat the present obesity epidemic.

\section{Introduction}

Obesity has increased at an alarming rate in recent decades, and it is currently estimated that between $25 \%$ and $30 \%$ of adults in countries such as the US and Australia are obese [1$3]$. Obesity is a major health issue as it contributes to conditions such as diabetes, cardiovascular disease, osteoarthritis, and some metabolic-related cancers $[3,4]$. Obesity has a multifactorial etiology comprising a range of genetic, metabolic, environmental, behavioral, and social/cultural factors [5]. However, the rapid rise in obesity suggests that the present epidemic is the result of recent social and environmental changes, with high fat diets and increasingly sedentary lifestyles identified as two of the main causes $[3,6]$.

There are, however, other factors that may be contributing to the present obesity epidemic which need to be addressed. One factor that is receiving increased attention is chronic sleep restriction ( $<7$ hours sleep a night). This is based on a number of recent epidemiological studies reporting that shorter sleep durations are associated with overweight and obesity in adults; see Cappuccio et al. [7] for a review. Some longitudinal data also indicate that short sleep predicts weight gain over a period of several years [8-12]. Furthermore, several laboratory-based studies have demonstrated that sleep restriction affects hormones involved in regulating energy balance, in a manner that is consistent with weight gain [13-18]. Interestingly, Chaput et al. [19] recently examined the relative contribution of nine risk factors for obesity (e.g., diet, physical activity, and sleep duration) over a six-year period. They found that sleep duration along with low calcium consumption and high disinhibition and restraint eating behavior significantly predicted weight gain; energy intake and physical activity were not significant predictors of weight gain.

These findings suggest that chronic sleep restriction could be a possible risk factor for obesity, and this may have implications for obesity management and prevention. However, our understanding of the relationship between 
chronic sleep restriction and obesity is incomplete as the processes through which chronic sleep restriction contributes to obesity, and the extent and magnitude of these effects, remain unclear. Furthermore, an important gap in the literature is that the causes, or determinants, of chronic sleep restriction have been largely overlooked. In this area of research, chronic sleep restriction has tended to be viewed as a result of behavioral sleep curtailment or an underlying sleep disorder [20]. This is a problem because the causes of chronic sleep restriction are likely to be complex and encompass many factors. These need to be identified and understood because they could influence the nature of the relationship between sleep and obesity and would likely have treatment implications.

Therefore, the purpose of the present paper is to integrate two important bodies of literature and examine (1) the determinants of chronic sleep restriction and (2) selected pathways linking chronic sleep restriction to obesity. This paper is not intended to provide a comprehensive review of these areas, as the relationship between chronic sleep restriction and obesity is likely to be complex and involve a multitude of processes. Instead, we focus on the factors and pathways that we feel are most important based on our assessment of the available literature. For example, we explore the following three potential pathways linking chronic sleep restriction to obesity: (1) neuroendocrine and metabolic pathways, (2) glucose regulation, and (3) waking behaviour.

It is also acknowledged that the association between chronic sleep restriction and obesity is likely to be bidirectional since obesity could also contribute to chronic sleep restriction (e.g., sleep apnea). However, this paper focuses primarily on chronic sleep restriction as a possible cause of obesity. We conclude the paper by presenting a schematic representation that integrates these pathways and provides an important framework that is currently lacking in the literature; this can be used to guide future research in this area.

\section{Determinants of Chronic Sleep Restriction}

Chronic sleep restriction is generally defined as habitual sleep durations that are less than 7 hours, but more than 4 hours, a night [21]. It is distinct from acute total sleep deprivation which refers to an absence of sleep over a minimum of 24 hours; this is neither a common nor chronic condition in humans. Chronic sleep restriction has become more common in recent decades, having more than doubled in the US since the 1960s $[22,23]$. Approximately onethird of adults in the US currently report chronic sleep restriction and similar figures have been observed in other countries [24-26]. Chronic sleep restriction is increasingly recognized as a health concern because it is associated with motor vehicle and industrial accidents, health conditions such as hypertension, diabetes, obesity, and depression, and increased mortality $[21,27,28]$.

Previous research investigating the link between chronic sleep restriction and obesity has not adequately addressed the underlying causes of chronic sleep restriction. Many studies have either overlooked the causes of chronic sleep restriction or viewed chronic sleep restriction as the result of voluntary sleep curtailment [20]. However, the determinants are likely to be complex and vary considerably between individuals. These need to be examined because the nature of the relationship between chronic sleep restriction and obesity could depend on, or vary according to, the precise cause of chronic sleep restriction. The potential to modify sleep duration to aid obesity prevention and management may also depend on the precise factor(s) that contribute to chronic sleep restriction. Therefore, the remainder of this section identifies some key determinants of chronic sleep restriction that require investigation in this context.

Sleep duration is influenced by a combination of genetic, health, sociodemographic, environmental, and behavioral factors; so there are likely a multitude of factors that could potentially contribute to chronic sleep restriction. Data from large-scale population studies have recently identified factors associated with chronic sleep restriction in adults at a population level. Most of these studies have been crosssectional, but they do provide an insight into the factors that could be important predictors of chronic sleep restriction. In particular, sociodemographic factors such as increased age, lower education level, lower income, ethnicity, and nonmarried status have been identified as strong predictors of chronic sleep restriction [24, 25, 29-31]. Health behaviors such as smoking, excessive alcohol consumption, lower levels of physical activity, increased television viewing, shift work, long working hours, and increased time commuting to and from work have also been associated with shorter sleep durations [24, 25, 30-34]. Several studies also demonstrate that physical and mental health status strongly predict sleep duration. For example, chronic diseases such as diabetes or cardiovascular disease $[24,25,29-31]$ and mental health conditions such as stress, depression, and sleep disorders (e.g., insomnia) have been associated with reduced sleep $[35,36]$. Dramatic changes in our physical environments in recent decades may also be contributing to chronic sleep restriction. For example, it is argued that we increasingly live in a 24-hour society that is characterized by more artificial light; this has the potential to disrupt natural circadian rhythms and adversely affect sleep [37].

There are, therefore, many sociodemographic, behavioral/lifestyle, health, and environmental factors that could contribute to chronic sleep restriction. Importantly, the precise causes of chronic sleep restriction are likely to vary considerably between individuals. Some individuals may voluntarily limit the amount they sleep in order to meet work, social, or family demands, whilst others engage in behaviors such as cigarette smoking and excessive alcohol consumption that adversely impact on sleep duration. In other individuals, an underlying health condition, medication use, or factors associated with a low socioeconomic status could be the primary cause(s) of chronic sleep restriction.

If chronic sleep restriction is to be targeted as a modifiable risk factor for obesity, there is a need to better understand the underlying factors contributing to chronic sleep restriction in different populations. This is important for two main reasons. First, the nature and magnitude of 
the relationship between chronic sleep restriction and obesity may depend on the underlying causes of chronic sleep restriction; this could influence the mechanisms or pathways linking chronic sleep restriction to obesity and also how susceptible an individual is to the effects of sleep restriction. Second, the potential to modify sleep through interventions may differ based on the specific cause(s) of chronic sleep restriction. For example, factors such as mental health problems and sleep disorders may require interventions from specialist practitioners. In contrast, factors such as work hours could be targeted by behavioral interventions that do not necessarily aim to reduce work hours (as this could have adverse consequences, such as a loss of income), but rather attempt to minimize the impact of work hours on sleep patterns. This could perhaps be achieved by addressing factors such as television viewing and time spent commuting to and from work which may be limiting sleep duration in people who work long hours $[33,34]$. Thus it is important that research investigating the relationship between chronic sleep restriction and obesity examines the determinants of chronic sleep restriction.

\section{Hypothesised Pathways Linking Chronic Sleep Restriction to Obesity}

There are many processes or pathways through which chronic sleep restriction could contribute to obesity and it is not feasible to address all of these in a single review. Instead we focus on the following three hypothesized pathways linking chronic sleep restriction to obesity: (1) neuroendocrine and metabolic pathways, (2) glucose regulation, and (3) waking behaviour. There is potential overlap between these pathways, but for the purposes of clarity, we discuss each of them separately.

The concept of energy balance is important in this context. A constant body weight depends on a balance between energy intake (diet) and energy expenditure (basal metabolic rate, physical activity, and thermogenesis). Under normal conditions energy balance is maintained by a complex regulatory system that involves multiple physiological pathways in the body which act on neural circuits to maintain body weight within a narrow range [38-40]. For example, the adipose tissue hormones leptin and adiponectin, the pancreatic hormone insulin, and the gastrointestinal hormones ghrelin, peptide $\mathrm{YY}_{3-36}$ (PYY), and glucagon-like peptide-1 (GLP-1) all act on hypothalamic circuits to influence energy balance. A chronic positive energy balance occurs when energy intake exceeds expenditure over a prolonged period of time; this has the potential to affect the processes involved in regulating body weight and can lead to obesity over time $[39,40]$.

\subsection{Hypothesis 1. Sleep Restriction Alters Neuroendocrine and} Metabolic Functioning. In a landmark series of experimental studies, Spiegel and colleagues [16-18, 41] demonstrated that short-term sleep restriction alters some neuroendocrine and metabolic hormones that are involved in the regulation of energy balance. In particular, six consecutive nights of sleep restriction (four hours sleep per night) were associated with increases in sympathetic nervous system (SNS) activity, evening cortisol levels and growth hormone levels (GH), and reductions in thyroid stimulating hormone (TSH), and leptin $[16,17,41]$. A follow-up study found that sleep restriction (four hours sleep per night) over two nights led to an $18 \%$ reduction in leptin and a $28 \%$ increase in ghrelin [18]. The increase in the leptin-to-ghrelin ratio corresponded with a $24 \%$ increase in hunger and a $23 \%$ increase in appetite that was mainly for energy dense foods. Other research groups have obtained similar results. For example, Guilleminault et al. [13] found that seven nights of sleep restriction (five hours sleep per night) led to a reduction in leptin rhythm amplitude. Schmid et al. [15] also found that a single night of 4.5-hour sleep led to an increase in ghrelin levels. Finally, Magee et al. [14] observed that two consecutive nights of five hours sleep led to a significant reduction in PYY levels and a corresponding decrease in satiety levels.

The profile of these hormonal changes is suggestive of increased energy intake, reduced energy expenditure and weight gain. For example, leptin, which is released in proportion to adipose tissue amount, acts on hypothalamic circuits to reduce energy intake and increase energy expenditure [42]. Insulin has many roles but also acts on hypothalamic circuits to reduce energy intake and increase energy expenditure $[42,43]$. The reductions in leptin and insulin observed with sleep restriction are therefore suggestive of increased food intake and reduced energy expenditure. Ghrelin is released primarily from the stomach when nutrient levels are low and acts on hypothalamic pathways to stimulate food intake $[44,45]$. The $\mathrm{PYY}_{3-36}$ molecule is released from the gastrointestinal tract in response to ingested nutrients and acts on the hypothalamus to reduce food intake [46, 47]. Therefore, the elevations in ghrelin and reductions in PYY observed with sleep restriction may be predictive of increased food intake.

Similarly, the increases in evening cortisol and GH levels may also be suggestive of weight gain. The elevation in $\mathrm{GH}$ levels observed by Spiegel et al. [41] was the result of an extended period of nocturnal GH secretion. This may have increased the amount of exposure of peripheral tissues to $\mathrm{GH}$; if prolonged this could impact on glucose regulation in a way that leads to obesity (this is discussed in more detail below) [41]. However, GH has been shown to promote lean tissue and reduce the accumulation of adipose tissue [48]. The precise implications of the increases in GH observed with sleep restriction are therefore unclear and require further investigation.

The elevations in cortisol following sleep restriction suggest greater activity of the hypothalamic-pituitary-adrenal gland (HPA) axis. This could reflect increased stress levels as the HPA axis plays an important role in regulating the stress response [49]. Importantly, elevated cortisol levels have been shown to promote increased food intake and the accumulation of visceral fat in humans [50]. Similarly, since TSH normally functions to stimulate basal metabolic rate, the reductions in TSH with sleep restriction are suggestive of a reduction in energy expenditure. 
The findings reviewed above indicate that short-term sleep restriction under controlled laboratory conditions alters neuroendocrine and metabolic hormones in a manner that is consistent with weight gain and obesity. However, we only have partial understanding of these mechanisms and there are a number of issues that remain to be addressed. First, it is not clear which brain mechanisms link sleep restriction with the observed alterations in metabolic and neuroendocrine functioning. The activation of the SNS with sleep restriction is one possibility since increased SNS activity inhibits the release of leptin from adipose tissue and may inhibit insulin release [51]. Increased SNS activity also inhibits vagal nerve activity, which could account for the rise in ghrelin level observed with sleep loss [52, 53]. However, increased SNS activity is typically associated with reductions in energy intake and increased energy expenditure over time [54]. Since this pattern is predictive of weight loss, increased activation of the SNS may not be the predominant mechanism through which sleep restriction alters energy balance and leads to weight gain. Instead, other mechanisms involving disruptions in the functioning of the suprachiasmatic nucleus (SCN) or activation of the HPA axis could be more important.

The SCN is located in the anterior hypothalamus and regulates the circadian rhythms of several physiological systems, including sleep, and the secretion of hormones involved in energy balance regulation [55]. Alterations in hormones such as leptin, cortisol, and TSH and GH observed with sleep restriction could therefore be the result of disrupted SCN output. As noted earlier, the increases in cortisol secretion observed with sleep restriction suggest increased activation of the HPA-axis, which is indicative of the stress response. The stress response serves an important adaptive purpose by supplying extra energy to body tissues in anticipation of a fight or flight response $[49,50,56]$. This leads to the release of cortisol which through a series of feedback loops signals the HPA axis to reduce cortisol secretion [57]. However, chronic or frequent stress can desensitise the HPA axis such that cortisol remains elevated; over time this can lead to an increase in visceral body fat since cortisol promotes fat accumulation and also inhibits the release of leptin $[49,50,58]$. Activation of the HPA axis may also explain the increased SNS activity observed with sleep restriction. Thus it is possible that the physiological changes observed with sleep restriction are the result of increased HPA axis activity and/or altered SCN output, which have a cascading effect on a number of physiological systems.

The second important consideration is that it is not clear whether the physiological effects of sleep restriction observed under laboratory conditions over a period of a few days are equivalent to prolonged (or chronic) sleep restriction as it occurs in free-living individuals. In particular, it is feasible to assume some degree of physiological adaptation to the effects of sleep restriction, but one can only speculate on the extent and nature of this adaptation. Moreover, the effects of sleep restriction as observed in laboratory-based settings may differ to the effects of chronic sleep restriction in free living adults. These are all important considerations because, as noted above, the causes of chronic sleep restriction could differ considerably between individuals and the impact of sleep restriction on energy balance may depend on the underlying cause of chronic sleep restriction. Finally, few studies have examined whether sleep restriction alters components of energy expenditure. Schmid et al. [59] demonstrated that sleep restriction led to a reduction in physical activity under free-living conditions, but there were no significant changes in food intake, hunger and appetite, and levels of leptin and ghrelin. This suggests that sleep restriction might alter energy expenditure but this requires further investigation in studies that also examine other components of energy expenditure such as basal metabolic rate or nonexercise activity thermogenesis. This is important because examining both sides of the energy equation (i.e., energy intake and expenditure) will be critical to understanding the processes through which sleep restriction promotes obesity.

\subsection{Hypothesis 2. Sleep Restriction Alters Glucose Regulation.} Another potential pathway linking chronic sleep restriction to obesity could involve disruptions in the regulation of glucose levels; this may also have implications for diabetes, which are discussed by Spiegel et al. [60]. Alterations in glucose regulation have been linked with weight gain and obesity. For example, Boulé et al. [61] found that lower blood glucose concentrations at the end of an oral glucose tolerance test (OGTT) predicted weight gain over a 6-year period. These results were explained according to the Glucostatic Theory of Appetite Control, which postulates that glucose plays an important role in the regulation of satiety and appetite [62]. In particular, reduced glucose utilization in important regions of the brain leads to perception of hunger and increased food intake, whereas higher glucose utilization in these same areas promotes a decrease in hunger and a cessation of eating [62].

Sleep restriction has been shown to affect glucose levels in humans. Spiegel et al. [17] found that six nights of sleep restriction led to a $30 \%$ reduction in glucose effectiveness (i.e., noninsulin dependent glucose utilization) and a $40 \%$ reduction in glucose utilization following intravenous glucose administration. These results have been supported by cross-sectional and prospective data. For example, Chaput et al. [63] found that habitually short sleepers had higher levels of fasting plasma glucose and insulin concentrations and lower blood glucose concentrations at the end of an OGTT. Chaput et al. [64] also found that individuals reporting short sleep had increased glucose area below fasting glucose concentrations; this is indicative of reactive hypoglycemia and predicted diabetes/impaired glucose tolerance at six-year followup.

Thus it is possible that chronic sleep restriction contributes to obesity by disrupting the regulation of glucose in a manner that promotes increased food intake. Chronic sleep restriction could potentially exert these effects via activation of the SNS or disruptions in hormones such as cortisol or GH [63]. As with the neuroendocrine pathways discussed above, there is a need for more longitudinal data examining whether prolonged sleep restriction does promote fat accumulation and lead to obesity by impairing glucose regulation. 


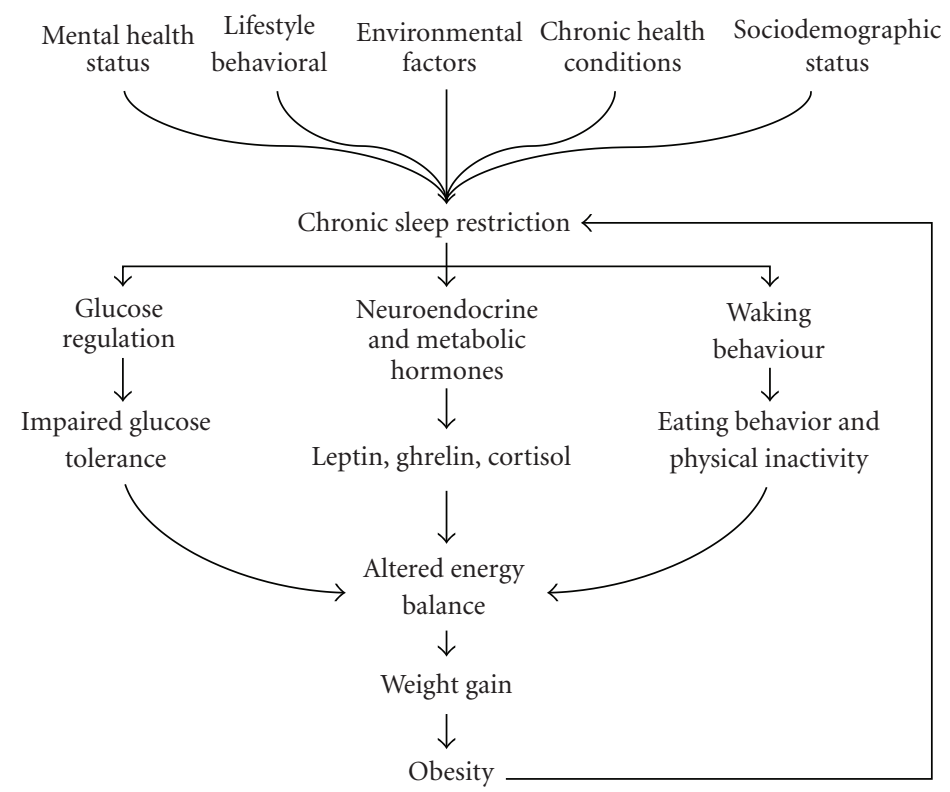

FIGURE 1: Schematic representation of the pathways linking chronic sleep restriction to obesity.

3.3. Hypothesis 3. Sleep Restriction Affects Waking Behaviour. The third hypothesis discussed in this paper is that chronic sleep restriction contributes to obesity by affecting waking behavior, and in particular promoting patterns of behavior that cause weight gain. It is well documented, for example, that consumption of food with a high-energy content and sedentary behavior (e.g., television viewing, physical inactivity) are strong risk factors for obesity [6]. Chronic sleep restriction could lead to obesity by promoting these behaviors and this has received some empirical support.

Nedeltcheva et al. [65] for example, recently examined the effects of 14 consecutive days of sleep restriction on food intake, energy expenditure, and neuroendocrine hormones. In contrast to the studies conducted by Spiegel and colleagues [16-18], which involved a mild form of calorie restriction, Nedeltcheva et al. [65] provided food to participants ad libitum. Their results indicated that sleep restriction led to an increase in calorie consumption that was attributed to snacking particularly during the night when the individual would normally have been sleeping (this is unlikely to reflect the night eating syndrome). Food intake during meal time remained unchanged with sleep restriction, as did energy expenditure. The findings suggest that short sleepers could be more susceptible to weight gain because they have more time to eat. The increase in consumption because of greater exposure to food (rather than increased hunger) suggests that in addition to the homeostatic factors reviewed above, nonhomeostatic factors may also be involved in the relationship between chronic sleep restriction and obesity [66]. Thus, future studies will need to further investigate both homeostatic and nonhomeostatic pathways linking chronic sleep restriction to obesity.

Another plausible behavioral pathway linking chronic sleep restriction to obesity involves fatigue, since individuals who get insufficient sleep are more likely to experience fatigue and daytime sleepiness [67]. It is possible that individuals engage in behaviors such as consumption of high-energy drinks or food to counter the effects of fatigue. Fatigue may also render individuals less likely to engage in physical activity $[68,69]$ and more likely to engage in sedentary behaviors such as television viewing. This pattern of behavior could also promote a positive energy balance and may partially account for the association between chronic sleep restriction and obesity.

\section{Integrating the Pathways Linking Chronic Sleep Restriction to Obesity}

Chronic sleep restriction may be an important risk factor for obesity and could have implications for obesity prevention and management. However, current understanding of the processes underlying the relationship between chronic sleep restriction and obesity is limited. The purpose of the present paper was not to provide a definitive review of the literature, but rather to integrate two important aspects of this relationship which are summarized in Figure 1. First, we examined potential determinants of chronic sleep restriction, which have been largely overlooked in the literature. We hypothesize that chronic sleep restriction could be the result of a range of factors including mental health status, lifestyle/behavioral factors, chronic disease, and sociodemographic status. It is important that these are examined further because the precise causes of chronic sleep restriction, and the potential to modify these in therapeutic settings, are likely to differ considerably between individuals. Second we examined the following three potential pathways through which chronic sleep restriction could potentially promote weight gain and obesity: (1) metabolic and neuroendocrine functioning, (2) glucose regulation, and (3) 
waking behaviour. These pathways have the potential to promote a positive energy balance and may explain the mechanisms linking chronic sleep restriction to obesity. The first challenge for researchers will be to demonstrate that chronic sleep restriction does impact on these and other pathways not examined in this paper. This is important because the effects of short-term sleep restriction may not correspond with the effects of chronic sleep restriction, as there may be some form of physiological adaptation over time. Thus, more long-term prospective studies examining the associations between changes in sleeping patterns, body composition, and the pathways identified in Figure 1 are needed. There is also a need to investigate whether the magnitude of the association between chronic sleep restriction and obesity, and the underlying pathways, varies according to the causes of chronic sleep restriction.

It should be noted that the association between chronic sleep restriction and obesity is likely to be bidirectional and circular, and this is depicted in Figure 1. Therefore, although the primary purpose of this paper was to review evidence indicating that chronic sleep restriction contributes to obesity, it is also possible that obesity contributes to chronic sleep restriction. For example, symptoms of obesity such as pain and discomfort and comorbid conditions such as obstructive sleep apnea have been shown to impair and disrupt sleep. As a result, there is a need for more experimental or prospective research to delineate the magnitude of the effect of chronic sleep restriction on obesity.

A final consideration is that it is not clear whether chronic sleep restriction can be modified through interventions, and whether these changes are effective in preventing and managing obesity. Research addressing these issues will be important in determining not only the pathways linking chronic sleep restriction to obesity but also whether chronic sleep restriction is a risk factor that can be modified to treat and prevent obesity. Currently there is a 12-month randomized controlled trial being conducted in the US (clinicaltrials.gov register number NCT00261898) that is examining whether increasing sleep duration in obese individuals who report short sleep affects body weight and other related variables (e.g., glucose regulation, neuroendocrine hormones). This study may provide clarification as to whether chronic sleep restriction can be targeted as a modifiable risk factor for obesity, but the results of this study have not yet been published. This area of research is significant given that the obesity epidemic continues to grow and poses a number of major health, social, and economic problems; targeting the amount we sleep could be an important step in combating this health problem.

\section{Disclosure Statement}

The authors report no financial conflicts of interest.

\section{References}

[1] A. J. Cameron, T. A. Welborn, P. Z. Zimmet, et al., "Overweight and obesity in Australia: the 1999-2000 Australian diabetes, obesity and lifestyle study (AusDiab)," Medical Journal of Australia, vol. 178, no. 9, pp. 427-432, 2003.
[2] C. L. Ogden, M. D. Carroll, L. R. Curtin, M. A. McDowell, C. J. Tabak, and K. M. Flegal, "Prevalence of overweight and obesity in the United States, 1999-2004," Journal of the American Medical Association, vol. 295, no. 13, pp. 1549-1555, 2006.

[3] World Health Organization, Obesity: Preventing and Managing the Global Epidemic, World Health Organization, Geneva, Switzerland, 2000.

[4] P. G. Kopelman, "Obesity as a medical problem," Nature, vol. 404, no. 6778, pp. 635-643, 2000.

[5] C. J. Stein and G. A. Colditz, "The epidemic of obesity," Journal of Clinical Endocrinology and Metabolism, vol. 89, no. 6, pp. 2522-2525, 2004.

[6] J. O. Hill, H. R. Wyatt, G. W. Reed, and J. C. Peters, "Obesity and the environment: where do we go from here?" Science, vol. 299, no. 5608, pp. 853-855, 2003.

[7] F. P. Cappuccio, F. M. Taggart, N.-B. Kandala, et al., "Metaanalysis of short sleep duration and obesity in children and adults," Sleep, vol. 31, no. 5, pp. 619-626, 2008.

[8] J.-P. Chaput, J.-P. Després, C. Bouchard, and A. Tremblay, "The association between sleep duration and weight gain in adults: a 6-year prospective study from the Quebec Family Study," Sleep, vol. 31, no. 4, pp. 517-523, 2008.

[9] J. E. Gangwisch, D. Malaspina, B. Boden-Albala, and S. B. Heymsfield, "Inadequate sleep as a risk factor for obesity: analyses of the NHANES I," Sleep, vol. 28, no. 10, pp. 12891296, 2005.

[10] G. Hasler, D. J. Buysse, R. Klaghofer, et al., "The association between short sleep duration and obesity in young adults: a 13-year prospective study," Sleep, vol. 27, no. 4, pp. 661-666, 2004.

[11] E. López-García, R. Faubel, L. León-Muñoz, et al., "Sleep duration, general and abdominal obesity, and weight change among the older adult population of Spain," American Journal of Clinical Nutrition, vol. 87, no. 2, pp. 310-316, 2008.

[12] S. R. Patel, A. Malhotra, D. P. White, D. J. Gottlieb, and F. B. $\mathrm{Hu}$, "Association between reduced sleep and weight gain in women," American Journal of Epidemiology, vol. 164, no. 10, pp. 947-954, 2006.

[13] C. Guilleminault, N. B. Powell, S. Martinez, et al., "Preliminary observations on the effects of sleep time in a sleep restriction paradigm," Sleep Medicine, vol. 4, no. 3, pp. 177$184,2003$.

[14] C. A. Magee, X.-F. Huang, D. C. Iverson, and P. Caputi, "Acute sleep restriction alters neuroendocrine hormones and appetite in healthy male adults," Sleep and Biological Rhythms, vol. 7, no. 2, pp. 125-127, 2009.

[15] S. M. Schmid, M. Hallschmid, K. Jauch-Chara, J. Born, and B. Schultes, "A single night of sleep deprivation increases ghrelin levels and feelings of hunger in normal-weight healthy men," Journal of Sleep Research, vol. 17, no. 3, pp. 331-334, 2008.

[16] K. Spiegel, R. Leproult, M. L'Hermite-Balerioux, G. Copinschi, P. D. Penev, and E. van Cauter, "Leptin levels are dependent on sleep duration: relationships with sympathovagal balance, carbohydrate regulation, cortisol, and thyrotropin," Journal of Clinical Endocrinology and Metabolism, vol. 89, no. 11, pp. 5762-5771, 2004.

[17] K. Spiegel, R. Leproult, and E. van Cauter, "Impact of sleep debt on metabolic and endocrine function," The Lancet, vol. 354, no. 9188, pp. 1435-1439, 1999.

[18] K. Spiegel, E. Tasali, P. Penev, and E. Van Cauter, "Brief communication: sleep curtailment in healthy young men is associated with decreased leptin levels, elevated ghrelin levels, and increased hunger and appetite," Annals of Internal Medicine, vol. 141, no. 11, pp. 846-850, 2004. 
[19] J.-P. Chaput, C. Leblanc, L. Pérusse, J.-P. Després, C. Bouchard, and A. Tremblay, "Risk factors for adult overweight and obesity in the quebec family study: have we been barking up the wrong tree," Obesity, vol. 17, no. 10, pp. 1964-1970, 2009.

[20] K. L. Knutson, K. Spiegel, P. Penev, and E. Van Cauter, "The metabolic consequences of sleep deprivation," Sleep Medicine Reviews, vol. 11, no. 3, pp. 163-178, 2007.

[21] D. F. Dinges, N. L. Rogers, and M. D. Baynard, "Chronic sleep deprivation," in Principles and Practice of Sleep Medicine, M. H. Kryger, T. Roth, and W. C. Dement, Eds., pp. 67-76, Elsevier Saunders, Philadelphia, Pa, USA, 4th edition, 2005.

[22] D. F. Kripke, L. Garfinkel, D. L. Wingard, M. R. Klauber, and M. R. Marler, "Mortality associated with sleep duration and insomnia," Archives of General Psychiatry, vol. 59, no. 2, pp. 131-136, 2002.

[23] D. F. Kripke, R. N. Simons, L. Garfinkel, and E. C. Hammond, "Short and long sleep and sleeping pills. Is increased mortality associated?" Archives of General Psychiatry, vol. 36, no. 1, pp. 103-116, 1979.

[24] P. M. Krueger and E. M. Friedman, "Sleep duration in the united states: a cross-sectional population-based study," American Journal of Epidemiology, vol. 169, no. 9, pp. 10521063, 2009.

[25] C. A. Magee, D. C. Iverson, and P. Caputi, "Factors associated with short and long sleep," Preventive Medicine, vol. 49, no. 6, pp. 461-467, 2009.

[26] A. Shankar, W.-P. Koh, J.-M. Yuan, H.-P. Lee, and M. C. $\mathrm{Yu}$, "Sleep duration and coronary heart disease mortality among Chinese adults in Singapore: a population-based cohort study," American Journal of Epidemiology, vol. 168, no. 12, pp. 1367-1373, 2008.

[27] P. Heslop, G. D. Smith, C. Metcalfe, J. Macleod, and C. Hart, "Sleep duration and mortality: the effect of short or long sleep duration on cardiovascular and all-cause mortality in working men and women," Sleep Medicine, vol. 3, no. 4, pp. 305-314, 2002.

[28] C. Hublin, M. Partinen, M. Koskenvuo, and J. Kaprio, "Sleep and mortality: a population-based 22-year follow-up study," Sleep, vol. 30, no. 10, pp. 1245-1253, 2007.

[29] J. Nunes, G. Jean-Louis, F. Zizi, et al., "Sleep duration among black and white Americans: results of the National Health Interview Survey," Journal of the National Medical Association, vol. 100, no. 3, pp. 317-322, 2008.

[30] K. A. Stamatakis, G. A. Kaplan, and R. E. Roberts, "Short sleep duration across income, education, and race/ethnic groups: population prevalence and growing disparities during 34 years of follow-up," Annals of Epidemiology, vol. 17, no. 12, pp. 948955, 2007.

[31] S. Stranges, J. M. Dorn, M. J. Shipley, et al., "Correlates of short and long sleep duration: a cross-cultural comparison between the United Kingdom and the United States," American Journal of Epidemiology, vol. 168, no. 12, pp. 1353-1364, 2008.

[32] T. Akerstedt, "Shift work and disturbed sleep/wakefulness," Occupational Medicine, vol. 53, no. 2, pp. 89-94, 2003.

[33] M. Basner and D. F. Dinges, "Dubious bargain: trading sleep for Leno and Letterman,” Sleep, vol. 32, no. 6, pp. 747-752, 2009.

[34] M. Basner, K. M. Fomberstein, F. M. Razavi, et al., "American time use survey: sleep time and its relationship to waking activities," Sleep, vol. 30, no. 9, pp. 1085-1095, 2007.

[35] R. M. Benca, "Mood disorders," in Principles and Practice of Sleep Medicine, M. H. Kryger, T. Roth, and W. C. Dement, Eds., pp. 1311-1326, Elsevier Saunders, Philadelphia, Pa, USA, 4th edition, 2005.

[36] Y. Dauvilliers, S. Maret, and M. Tafti, "Genetics of normal and pathological sleep in humans," Sleep Medicine Reviews, vol. 9, no. 2, pp. 91-100, 2005.

[37] S. M. W. Rajaratnam and J. Arendt, "Health in a 24-h society," The Lancet, vol. 358, no. 9286, pp. 999-1005, 2001.

[38] M. W. Schwartz, S. C. Woods, D. Porte Jr., R. J. Seeley, and D. G. Baskin, "Central nervous system control of food intake," Nature, vol. 404, no. 6778, pp. 661-671, 2000.

[39] B. M. Spiegelman and J. S. Flier, "Obesity and the regulation of energy balance," Cell, vol. 104, no. 4, pp. 531-543, 2001.

[40] B. E. Wisse, F. Kim, and M. W. Schwartz, "Physiology: an integrative view of obesity," Science, vol. 318, no. 5852, pp. 928-929, 2007.

[41] K. Spiegel, R. Leproult, E. F. Colecchia, et al., "Adaptation of the 24-h growth hormone profile to a state of sleep debt," American Journal of Physiology, vol. 279, no. 3, pp. R874-R883, 2000.

[42] D. Porte Jr., D. G. Baskin, and M. W. Schwartz, "Leptin and insulin action in the central nervous system," Nutrition Reviews, vol. 60, no. 10, pp. S20-S29, 2002.

[43] X.-F. Huang, X. Xin, P. McLennan, and L. Storlien, "Role of fat amount and type in ameliorating diet-induced obesity: insights at the level of hypothalamic arcuate nucleus leptin receptor, neuropeptide $\mathrm{Y}$ and pro-opiomelanocortin mRNA expression," Diabetes, Obesity and Metabolism, vol. 6, no. 1, pp. 35-44, 2004.

[44] A. M. Wren, L. J. Seal, M. A. Cohen, et al., "Ghrelin enhances appetite and increases food intake in humans," Journal of Clinical Endocrinology and Metabolism, vol. 86, no. 12, pp. 5992-5995, 2001.

[45] J. M. Zigman and J. K. Elmquist, "Minireview: from anorexia to obesity-the yin and yang of body weight control," Endocrinology, vol. 144, no. 9, pp. 3749-3756, 2003.

[46] R. L. Batterham, M. A. Cowley, C. J. Small, et al., "Gut hormone PYY3-36 physiologically inhibits food intake," Nature, vol. 418, no. 6898, pp. 650-654, 2002.

[47] X.-F. Huang, M. Han, and L. H. Storlien, "The level of NPY receptor mRNA expression in diet-induced obese and resistant mice," Molecular Brain Research, vol. 115, no. 1, pp. 21-28, 2003.

[48] D. E. Berryman, E. O. List, D. T. Kohn, K. T. Coschigano, R. J. Seeley, and J. J. Kopchick, "Effect of growth hormone on susceptibility to diet-induced obesity," Endocrinology, vol. 147, no. 6, pp. 2801-2808, 2006.

[49] O. Van Reeth, L. Weibel, K. Spiegel, R. Leproult, C. Dugovic, and S. Maccari, "Interactions between stress and sleep: from basic research to clinical situations," Sleep Medicine Reviews, vol. 4, no. 2, pp. 201-219, 2000.

[50] P. Björntorp, "Do stress reactions cause abdominal obesity and comorbidities?” Obesity Reviews, vol. 2, no. 2, pp. 73-86, 2001.

[51] D. V. Rayner and P. Trayhurn, "Regulation of leptin production: sympathetic nervous system interactions," Journal of Molecular Medicine, vol. 79, no. 1, pp. 8-20, 2001.

[52] R. B. Heath, R. Jones, K. N. Frayn, and M. D. Robertson, "Vagal stimulation exaggerates the inhibitory ghrelin response to oral fat in humans," Journal of Endocrinology, vol. 180, no. 2, pp. 273-281, 2004.

[53] D. L. Williams, H. J. Grill, D. E. Cummings, and J. M. Kaplan, "Vagotomy dissociates short- and long-term controls of circulating ghrelin," Endocrinology, vol. 144, no. 12, pp. 5184-5187, 2003. 
[54] E. Ravussin and P. A. Tataranni, "The role of altered sympathetic nervous system activity in the pathogenesis of obesity," Proceedings of the Nutrition Society, vol. 55, no. 3, pp. 793-802, 1996.

[55] C. B. Saper, J. Lu, T. C. Chou, and J. Gooley, "The hypothalamic integrator for circadian rhythms," Trends in Neurosciences, vol. 28, no. 3, pp. 152-157, 2005.

[56] P. Björntorp and R. Rosmond, "Obesity and cortisol," Nutrition, vol. 16, no. 10, pp. 924-936, 2000.

[57] I. Hindmarch, "Examining the horizons of depression: beyond the monoamine model," Human Psychopharmacology, vol. 16, pp. 203-218, 2001.

[58] M. F. Dallman, N. Pecoraro, S. F. Akana, et al., "Chronic stress and obesity: a new view of "comfort food"'" Proceedings of the National Academy of Sciences of the United States of America, vol. 100, no. 20, pp. 11696-11701, 2003.

[59] S. M. Schmid, M. Hallschmid, K. Jauch-Chara, et al., "Shortterm sleep loss decreases physical activity under free-living conditions but does not increase food intake under timedeprived laboratory conditions in healthy men," American Journal of Clinical Nutrition, vol. 90, no. 6, pp. 1476-1482, 2009.

[60] K. Spiegel, K. Knutson, R. Leproult, E. Tasali, and E. Van Cauter, "Sleep loss: a novel risk factor for insulin resistance and type 2 diabetes," Journal of Applied Physiology, vol. 99, no. 5, pp. 2008-2019, 2005.

[61] N. G. Boulé, J.-P. Chaput, E. Doucet, et al., "Glucose homeostasis predicts weight gain: prospective and clinical evidence," Diabetes/Metabolism Research and Reviews, vol. 24, no. 2, pp. 123-129, 2008.

[62] J.-P. Chaput and A. Tremblay, "The glucostatic theory of appetite control and the risk of obesity and diabetes," International Journal of Obesity, vol. 33, no. 1, pp. 46-53, 2009.

[63] J.-P. Chaput, J.-P. Després, C. Bouchard, and A. Tremblay, "Association of sleep duration with type 2 diabetes and impaired glucose tolerance," Diabetologia, vol. 50, no. 11, pp. 2298-2304, 2007.

[64] J.-P. Chaput, J.-P. Després, C. Bouchard, A. Astrup, and A. Tremblay, "Sleep duration as a risk factor for the development of type 2 diabetes or impaired glucose tolerance: analyses of the Quebec Family Study," Sleep Medicine, vol. 10, no. 8, pp. 919-924, 2009.

[65] A. V. Nedeltcheva, J. M. Kilkus, J. Imperial, K. Kasza, D. A. Schoeller, and P. D. Penev, "Sleep curtailment is accompanied by increased intake of calories from snacks," American Journal of Clinical Nutrition, vol. 89, no. 1, pp. 126-133, 2009.

[66] C. B. Saper, T. C. Chou, and J. K. Elmquist, "The need to feed: homeostatic and hedonic control of eating," Neuron, vol. 36, no. 2, pp. 199-211, 2002.

[67] J. S. Durmer and D. F. Dinges, "Neurocognitive consequences of sleep deprivation," Seminars in Neurology, vol. 25, no. 1, pp. 117-129, 2005.

[68] R. C. Brownson, E. A. Baker, R. A. Housemann, L. K. Brennan, and S. J. Bacak, "Environmental and policy determinants of physical activity in the United States," American Journal of Public Health, vol. 91, no. 12, pp. 1995-2003, 2001.

[69] H. E. Resnick, E. A. Carter, M. Aloia, and B. Phillips, "Crosssectional relationship of reported fatigue to obesity, diet, and physical activity: results from the third national health and nutrition examination survey," Journal of Clinical Sleep Medicine, vol. 2, no. 2, pp. 163-169, 2006. 


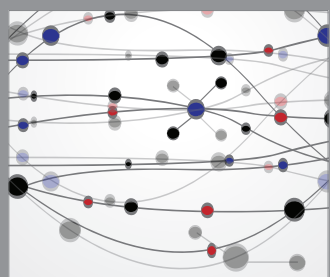

The Scientific World Journal
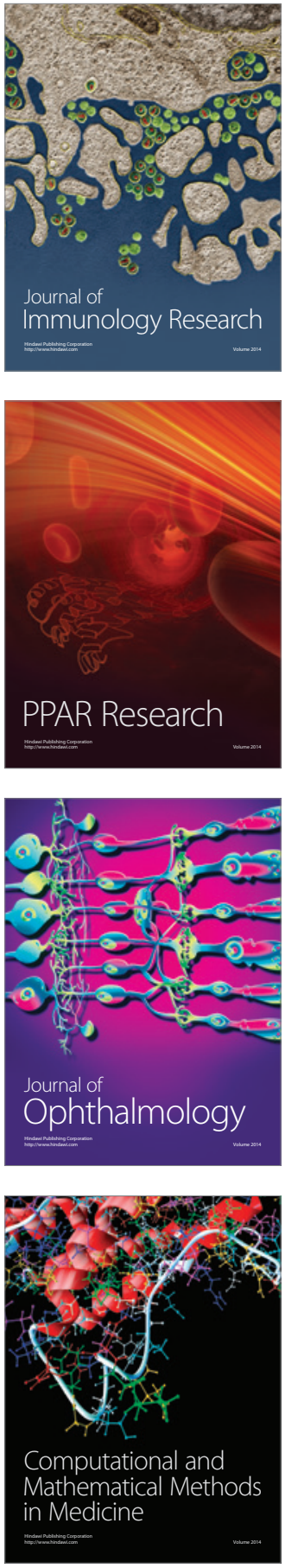

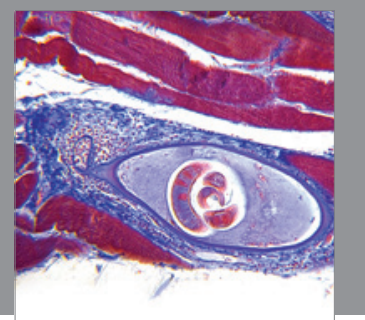

Gastroenterology

Research and Practice
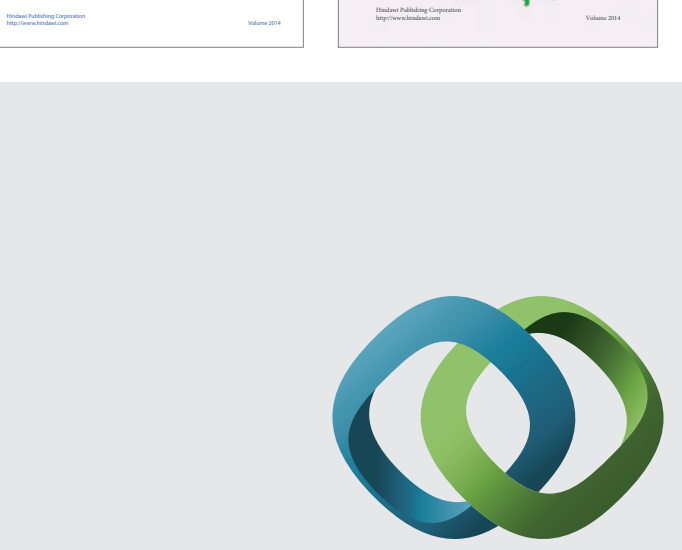

\section{Hindawi}

Submit your manuscripts at

http://www.hindawi.com
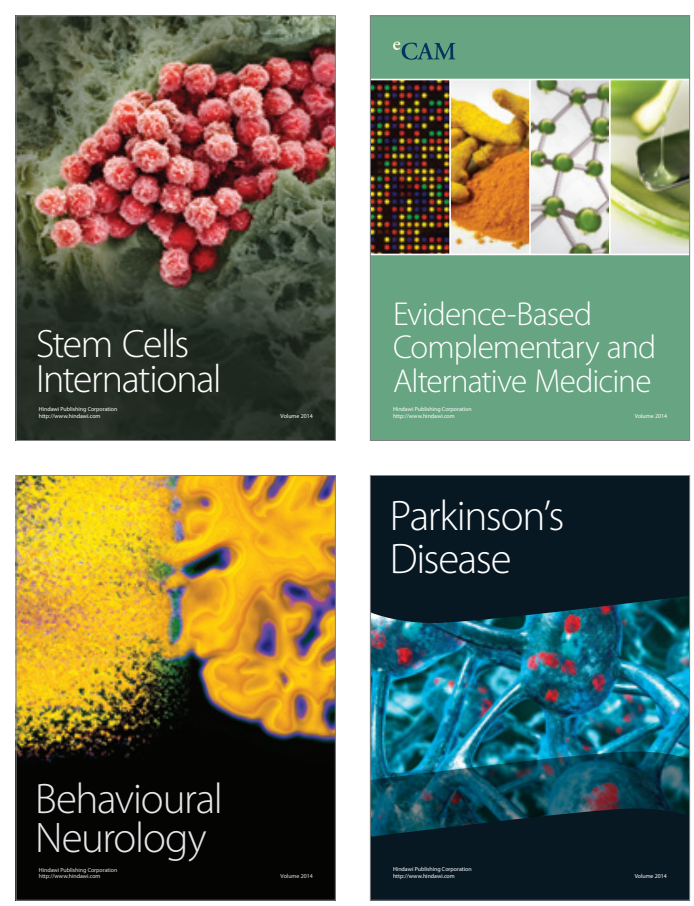

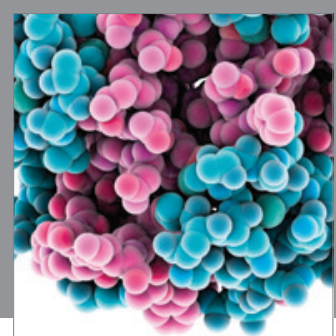

Journal of
Diabetes Research

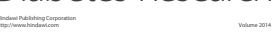

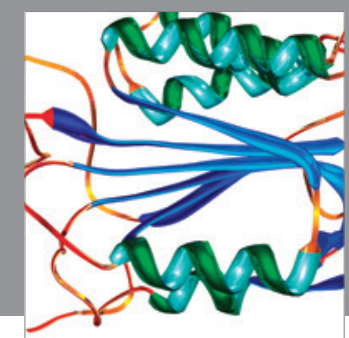

Disease Markers
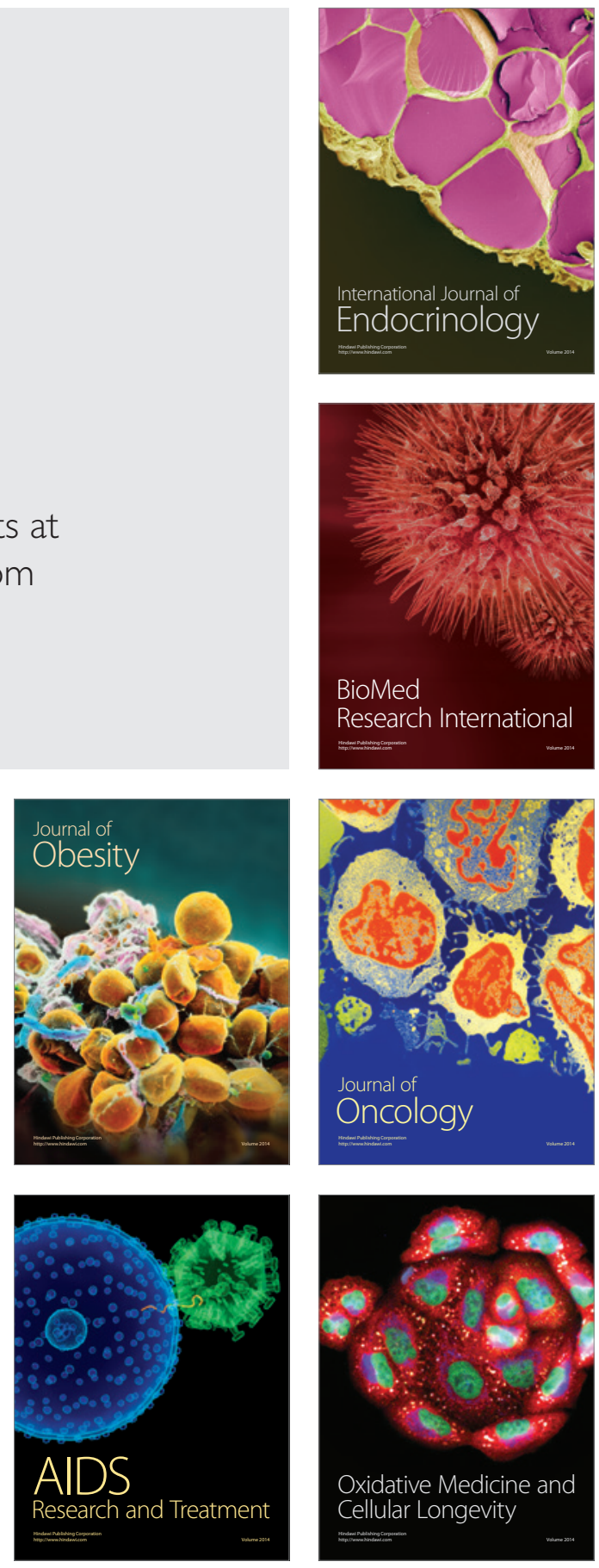